\title{
Cloud service purchase decision process
}

\author{
Jaromir Veber
}

\begin{abstract}
This article analyzes questions arising from a choice of available cloud computing services. Precisely what type of services should an organization choose? The article aims to introduce decision-making process according to the best practices. In order to describe this decision-making process we divide organizations into classes and for each class we recommend one or more available types of suitable cloud computing services. Moreover, there are introduced interesting cloud computing provider practices and service aspects including service operation, service parameters and service costs There are also introduced steps for customers how to deal with mentioned aspects.
\end{abstract}

Index Terms - cloud computing, decision making, risk analysis

\section{INTRODUCTION}

$\mathrm{C}$ urrent prevailing economic crisis affects considerable amount of organizations, and very often squeeze their revenues. Frequently used way to tackle this unfavorable economic development is to cut costs as described by Hoadley in [5]. This pressure affects all departments of organization including information and communications technology (ICT) department.

As a result, in the last few years and the near future the main task of ICT managers is cost cutting. Chief information officers (CIO) need to solve a strategic issue: "how to ensure operations of ICT department with lower costs?"

Undoubtedly, numerous methodologies focused on ICT management are on hand. These allow effective management of allocated resources; listed for example in Van Grembergen [10] or Doucek [4].

However, there is different approach to coping with the efficiency problem; deployment of new technologies and outsourcing. Solution introduced in [1], evolved over the last few years and offers advantage of both aforementioned trends, called Cloud Computing (CC). American National institute of Standards and Technology (NIST) defines cloud computing as:

"Cloud computing is a model for enabling ubiquitous, convenient, on-demand network access to a shared pool of configurable computing resources (e.g., networks, servers, storage, applications, and services) that can be rapidly provisioned and released with minimal management effort or service provider interaction." [7]

Interest in cloud computing among enterprises most often stems from the need to reduce costs associated with the use of ICT (Information and communications technology) services. This demand is often satisfied by cloud computing solution, as the cloud supply is offered at reasonable prices, it provides the opportunity for monitoring service usage and the financial flows into ICT are transparent for organizational senior management.

$\mathrm{CC}$ services differ from complex outsourcing. Main difference is in specific relationship between customer and provider. There's distance in communication between provider and customer with $\mathrm{CC}$. Term $\mathrm{CC}$ covers relatively wide range of services that have some same features (for example pay-asyou-go system) yet there may be significant differences between particular CC services.

Whether and how to use CC services is definitely demanding managerial decision and the position of responsible employee in at the executive level, mostly CIO. The most common scenario is that the cloud computing solution purchase is triggered by top management looking for cost savings in ICT. As a response, the CIO requests a study of possible cloud computing usage conducted internally or externally. It is a requirement that $\mathrm{CC}$ solution is matching company standard of quality and response time for ICT services. Furthermore, the study involves calculation and comparison of current and considered CC solution expenses. The results of such study are inputs for decision ultimate decision about CC purchase.

The question in this case is "What kind of services to use or whether to use them at all?" It is answered either by ICT employee or by CIO. The final decision is most frequently endorsed by senior management of the company.

\section{PROBLEM FORMULATION}

Conducting a technical study describing possible usage of cloud computing services requires processing and analyzing of numerous information about available services. Technical analysis is often time consuming and therefore it is tempting to skip some essential considerations or to omit some important steps. Such omissions would lead to shortened and wrongly concluded study and consequently wrong decision about future strategy of organization may be made.

The goal of this article is to introduce processes, which will help during selection period of cloud services type and ease the authorized person a decision about using $\mathrm{CC}$ considering all relevant factors and risks involved in this decision.

\section{DECISION POCESS}

Full decision process has three steps:

1. Chose possible class of cloud computing services

2. Check market for available offerings of this type of cloud services

3. Choose one offering from available offers 
During decision process in first step: "what class of cloud services to use?" Available cloud service classes are described in part VI. We have to proceed from customer initial situation. The decision depends on the business situation, or more precisely, the business situation of ICT. Therefore, we need to classify customers into distinct classes, where each customer class will have different needs for ICT services. The initial situation of customer from our point of view depends on volume of provided ICT services that we can easily observe as the size of ICT department. Customer classification process is described in part VII.

Second interesting standpoint is associated with the age of organization. Especially newly established organizations are eligible for cloud adoption.

Second step is dependent on common market research that does not need special guidance, thus is not covered by this article.

Third step is the most difficult one. The part VIII of the article should help with this step mentioning operationaleconomic risks involved in service selection. There are of course other risks involved - for example often mentioned security risks and legal risks. However, these topics are covered by work of numerous other authors for example [12, $11,13,14]$ unlike economical and operational risks.

\section{PREVIOUS WORK}

Similar problem was solved in an article "Selecting the right Cloud" published by David Linthicum [6]. However this article was published in 2009 and was focused on well-known service portfolio known until 2009 (mostly Amazon services).

In 2010, the global consortium OpenGroup published an article "Cloud Buyers' Decision Tree" [9]. This article introduced likely practical approach for decision about moving into cloud either private, public or not using cloud at all. I found this approach general and consider it difficult for practical deployment.

Adam Goldstein from Dartmouth university published an article "Calculating the Cloud: Determining the True Cost of Hosting Servers in the Cloud" [3]. This article is practical, however focused only on infrastructure cloud services.

Most articles focused on cloud computing risks contain information about security and privacy risks and connected legal implications. However, economic and operational part of risks is unfortunately left out so far, that is why these risks are discussed and emphasized in this article.

\section{Metodology}

Foundation for this article is completed research lead by author of the article in the Czech Republic. Therefore, it is relevant regionally and recommendations for other regions may vary in some aspects.

The research was conducted in a form of discussions (with research participants). Research involved two different parties: cloud service providers and cloud service customers.
Dialogue with providers was focused on aspects of provided services. What kind of customers do the providers look for with offered services and why? What is the price of the services and how is it calculated? Additionally, we asked for the detailed description of provided services and comparison to other similar services provided by other providers was demanded.

The dialogue with (potential) customers was focused on their ICT demands. What services are the ICT departments managing? Are the organizations interested in cloud services? What do the customers expect from cloud service? What are the main advantages and disadvantages of current cloud service solution?

The participants of this research were employees (top company management or CIO) from approximately twenty organizations (about half were smaller organizations or entrepreneurs):

Cloud providers: Cloud4.com, IBM, SAP, Eltodo, Oracle.

Cloud customers: Deloitte (consulting), Ceska Pojistovna (insurance), Vseobecna fakultni nemocnice v Praze (hospital), Elektrizace Zeleznic (railway projects), Metrostav (construction), and others.

Subsequently additional internet published data about cloud offer were collected. A comparison of data obtained for each cloud offering type was performed using collected data. The result of this comparison was an evaluation of individual aspects mentioned below:

- $\quad$ significant economic items that depend on the use of service or are somehow hidden to customer,

- $\quad$ the most frequently utilized model of payment for the service,

- dependence on providers and the resulting risks,

- the parameters that determine the quality and service.

\section{AVAILABLE CLOUD SERVICES}

The question is "What classes of cloud computing may an organization employ?" Above mentioned NIST [7] define following deployment models:

- $\quad$ Private cloud,

- Community cloud,

- $\quad$ Public cloud,

- Hybrid cloud,

- Virtual private cloud.

There are defined well-known cloud service models as well:

- $\quad$ Cloud Software as a Service (SaaS).

- $\quad$ Cloud Platform as a Service (PaaS).

- Cloud Infrastructure as a Service (IaaS).

\section{A. Private cloud}

Solution based on private cloud [7] - "The cloud infrastructure is operated solely for an organization. It may be managed by the organization or a third party and may exist on premise or off premise." 
That means it is mostly operated by either local organization specialized on cloud computing (local provider) or by customer himself.

The advantage of privately provided cloud is better control over ICT security and better oversight over provided services.

This solution is mostly not as cost effective as public cloud (described below) but may allow customer better management and flexibility than "as is" ICT.

Provided service models for private cloud are mostly IaaS. Private cloud means a lot of possible providers and much more available SLAs. Private cloud is mostly reachable through fast optical or microwave lines so the throughput and latency should satisfy all customers.

\section{B. Public Cloud}

Public cloud means [7] - "The cloud infrastructure is made available to the general public or a large industry group and is owned by an organization selling cloud services".

There are two distinct types of provider for this type of cloud:

1. Global providers (Amazon, Google, Microsoft ...)

2. Local/regional providers (using available data centers in customers' country)

Global providers use their own data centers, mostly have their own cloud software and provide better prices.

Local providers on the other hand rent space in data centers (managed by third party) and use some kind of virtualization and cloud software (cloud software listed in [8]) to provide services to their own customers.

Global providers may provide many different service models and they provide them to all customers with common SLAs (customization of SLAs is done only for sizable customers). They may provide cloud services to thousands of customers.

Local providers manage services only to tens of customers maybe to hundreds. That is why they are able to provide customized SLA on demand to satisfy customers' needs. They provide mostly only IaaS.

\section{Hybrid cloud}

This type of solution is a combination of private and public cloud connected by cloud software so it's not exactly specific type but much more extension to available cloud services. Therefore, some services are provided in public part of cloud and sensitive data with high requirements on control and security are processed in private part of cloud.

Cloud solution based on hybrid cloud is not common in our region. It may be ideal solution for some customers for specific (for example banking) services but in general, classification it is not recommended later in the article.

\section{Community cloud}

This type of solution is mentioned in [2] and it is a specific type of cloud managed and owned by community. Communities include various organizations that are able to cooperate on democratic principles.

\section{E. Virtual private cloud}

Is a cloud based on creating own private network inside public cloud using VPN (Virtual Private Network). The private network allows better control over IP address range and subnets. Connection to cloud is automatically secured.

\section{F. IaaS}

Virtual infrastructure offers based on cloud computing are available as private cloud services as well as public cloud. These two approaches differ in the way of sharing hardware. In the case of private cloud infrastructure is dedicated to one customer solely, while the public cloud, customers share available computational resources.

Provider is providing virtual machines with defined parameters to customers.

\section{G. PaaS}

PaaS services are designed for running and developing cloud applications. Such applications may be available from around the world and the development itself should be very agile thanks to well-designed API. For the operation and development of service on PaaS platform a specific development environment and server to run developed applications is offered.

\section{H. SaaS}

SaaS services are available for many years, their offer are a few years older than the first references about cloud computing. Nevertheless, a significant development and wide availability of SaaS offers is possible only in recent years with the development of cloud computing.

\section{CUSTOMER CATEGORIZATION AND SERVICE CLASS RECOMENDATION}

The decision process does not depend so much on a size of organization but on a size of its ICT. For the purposes of this research author in cooperation with organizations involved defined categories depending on an extent of hardware (servers) used in ICT department. This number of course depends on number of ICT services that an organization uses and demanded capacity for such services. For this classification, three different ICT department classes were defined:

\section{- $\quad$ Large-scale ICT (more than 60 servers). \\ - $\quad$ Midsized ICT (8-60 servers). \\ - $\quad$ Small ICT (0-7 servers).}

It is necessary to count virtual servers if virtualization is employed in considered ICT. There may be a discussion about specific values used to distinguish the classes. The borders cannot be exact (because every organization use ICT department in different way and the management of ICT may choose different approach for server usage) so the aforementioned ICT department classification may enable one ICT department class to be eligible for two classes. 


\section{A. Large-scale ICT}

\section{1) Characterization}

Customers owning Large-scale ICT are making huge investments into it. If they operate the ICT by themselves, it means high requirements for service and of course burden for organization management that have to manage it as well. Such an ICT structure contains many custom applications developed either by organization itself or by some external provider developing specific software. Some kind of customized ERP software will usually be in place. Such applications are not easily available as SaaS service and developing them immediately as PaaS is not common.

\section{2) Recommendation and choice of strategy}

Customers owning Large-scale ICT are often using some kind of virtualization with transition to private cloud solutions (sometimes virtual private cloud). Either "in-house", where the provider provides operation of cloud and customer got his own cloud on site or "hosted", where both locations and operation is managed by provider, however customer does not share hardware with other customers.

Although private cloud is usually more expensive than public cloud, in the case of employment by large-scale ICT, the costs may be lower and operation transparent and flexible. The organizational ICT service parameter demands need to be specified in SLA (service level agreement) concluded between customer and provider. The principles and service levels of an SLA are in most cases enforced much better from external provider than form internal ICT department. Organizations owning really huge ICT - 1000 servers or more are often satisfied by virtualization only and they don't benefit much from cloud computing (except some special cases of organizations that have dynamically changing ICT demands). The costs of this approach are similar to private cloud so decision of these organizations depends mostly on importance of other smaller benefits brought by private cloud (better automation, operational costs, flexibility and less strain for management).

If such an organization is a part of holding, group or some kind of chain (e.g. retail chain, manufacturing chain ...) there is an opportunity arising to establish a community cloud. In community cloud, the customers share cloud, however all cloud users are known and data exchange between users is desirable and efficient.

Customers from large-scale ICT category are using many application services that are hard to find on SaaS market, so even though they can use some SaaS services they cannot just throw away all hardware and use only SaaS services. However, the possibility of developing new services on platform (PaaS) is interesting target for this customer group and all projects containing some application development should consider PaaS as an option.

\section{B. Midsized ICT}

1) Characterization

Most frequently used types of software by midsized ICT departments are:

-

- mail software (MS Exchange),

- $\quad$ file server software (for data sharing),

- web server software,

- accounting software,

- $\quad$ organization customized software (database and its user interface),

- $\quad$ CRM software.

Owners of midsized ICT structures are often using some kind of ERP software (with some customization). This software provides support for organization key processes. However, there are other systems in usage. If these are not part of ERP, organization needs some country specific accounting software and many organizations use some kind of mail software too. Moreover, when the organization is customer oriented it should have some kind of CRM software on the top of it. To support sharing and exchange of documents organizations mostly use file servers with some kind of NAS (network area storage) support. Many organizations use a web-server however; this kind of server is very often hosted locally. Many organizations with midsized ICT have some custom applications developed either by some external subjects or by themselves - these applications are often database based.

\section{2) Recommendation and choice of strategy}

Midsized ICT does not require as high investments as largescale ICT and therefore complete movement into private cloud is not the right decision, because it will not lead in most cases to reduction of costs. Companies providing private cloud solutions are calculating price depending on the number of server racks used by customer and midsized ICT hardly fills two racks. If the company does not utilize full rack, the unused rack capacity is charged by the provider anyways.

For midsized ICT there is still possibility to employ IaaS and use servers almost the same way on virtualized infrastructure after cloud as before cloud. However, this group of customers' demands reliable public cloud with customer specific SLA. Global providers' SLAs are usually not sufficiently tailored to the needs of midsized ICT companies. However it is possible to try local or regional) cloud providers. Local providers are using local data centers and due to this fact, the response times are sufficient. Local provider does not have so many customers so they are able to alter SLA and service price to fit customer needs. Local providers are fully familiar with local law so there should be no legal complication with usage of their cloud. Virtual private cloud offer is of course very good fit for this customer group however, it is necessary to satisfy connection speed and response time's needs.

Most of the cloud services used by midsized ICT should be available in form of SaaS. Some ERP developers are working or providing "on-demand" services as well. On-demand ERP lacks customization so not all customers would be satisfied with this option. On the other hand, this offer is still very new 
and quickly developing, thus in the future SaaS services with customization may be available in ERP. CRM was the first kind of software widely available in form of SaaS. Other systems like E-mail and file sharing services (in SaaS form) were introduced by both Microsoft and Google as well as by other minor providers, so these are available too.

Accounting software is very often provided by local software developers, because it is influenced by legal system. Some of local developers have found the market niche and they are developing their own SaaS solutions.

Second possible way for midsized ICT is to use SaaS only but this choice leads to high dependence on SaaS providers moreover, not all services required might be available in form of SaaS.

\section{Small ICT}

\section{1) Characterization}

ICT department of an organization with small ICT is mostly operated by one employee or by an outsourced professional. Organizations owning small ICT would preferably own no ICT at all (in this case, we mean servers, software and specialists to manage it all). They are not using any customized ERP software and the role of such software is provided by either office software or small database system applications.

2) Recommendation and choice of strategy

The transit into public cloud is not such an obstacle for organization owning small ICT and that is because of much lower dependence on ICT services. This is why most of small ICT customers welcome services in form of SaaS. They demand working helpdesk by provider and software that actually works without any maintenance. This way, they can close down internal ICT department completely. So small ICT department is typical adept for transition to SaaS cloud services.

\section{Recently established organizations and new bureau}

\section{1) Characterization}

This type of organizations has an advantage in cloud adoption no matter what the size of planned ICT support is. Business processes are not completely formed and demands on ICT are not so specific. To support newly formed business processes the cloud provide great flexibility that is necessary for recently established organization.

\section{2) Recommendation and choice of strategy}

Organization should try available SaaS offers and develop its own custom applications on PaaS.

In case of new bureau with specific software needs, there is still possibility to satisfy these needs and take an advantage of fast deployment due to (probably locally provided) IaaS, before ICT needs are stabilized.

\section{OPERATIONAL-ECONOMIC RISKS OF DIFFERENT CLOUD SECVICE CLASSES}

\section{A. Private cloud at the customer}

\section{1) Service description}

Private cloud can also be operated in such a way that the hardware is physically stored on customer's site. In this case, it is also possible that the customers buy their own hardware that means investment costs in order to increase the company value. Implementation in this case is usually realized by contract with a fixed monthly fee, the customer gets a certain number of physical machines placed inside the customer's own "server room"). Using more physical machines also means higher price. A customer can change the requirements for the number of machines but with low flexibility (change once a month or six months). Requirements for performance improvements are often made quicker by providers (within days).

\section{2) Service parameters}

The performance of each virtual machine depends on the hardware and the virtualization settings (that is laid down by the customer). The parameters can be changed only through the exchange of hardware.

\section{3) Economic characteristics}

In terms of deployment and the resulting price, this method is very similar to the method of managing own ICT department (partly outsourced). Therefore operating-economic or security risks are same as in case of managing own ICT services. The customer's monthly fee covers the rental of hardware, software, and cloud machine maintenance. The costs of energy consumption (including cooling) and supervision remains the responsibility of the customer. Although ICT operating costs are not so different from operating own ICT, the investment costs may be spread over a longer period (it's possible to avoid the disposable investment in hardware, etc.). Price depends on the number of machines, machine configuration, and choice of accessories such as disk storage. In this case, the price is very often fixed by agreement between the provider and the customer.

\section{B. Private cloud at the provider}

\section{1) Service description}

Hardware is placed at the provider's site and he ensures oversight over hardware operation, cooling and electricity supply. The customer is connected directly to his hardware using usually dedicated optical line. It is also possible that the hardware is either owned by the customer (so the price may be reflected in the investment costs) or by service provider (so the price is reflected in the operating costs). Requirement changes in the number of machines or reconfigurations are possible, while the flexibility in this case often depends on the flexibility of the provider. Some of them offer change within minutes, some within months (increasing performance is often done with better response than decreasing).

2) Service parameters

The performance of each virtual machine depends on the 
hardware and the virtualization settings (that is laid down by the customer). Parameters are changed only through change of hardware. Providers should therefore ensure within the SLA, that the hardware would not change. Change should either mean better parameters or be part of a new SLA.

\section{3) Economic characteristics}

Payment in this case is also dealt through a monthly payment and price depends on the number of machines. In comparison to the above-mentioned placement at the customer, the price is often lower because the provider can better optimize the cost of security, cooling and maintenance. In addition to monthly fee for depositing machines, the extra charge for electricity consumed by the machines is paid. At this point, each provider may vary in calculation mechanism of the charged fee, as some install wattmeter deducting directly consumed energy, while others statically count the energy consumed by the formula, according to the nominal value of consumption written on the label on the hardware resources and time that the machine was running. Therefore, the resulting price for consumed energy with identical hardware placed at different providers may vary greatly and it is important to watch closely this part of concluded contract.

\section{Public cloud IaaS}

\section{1) Service description}

Sharing of virtualized machines allows efficient sharing of hardware resources for providers therefore; they may offer lower prices in comparison to private cloud services. In this case, the customer receives a virtual machine with defined parameters. However, in this case, different customers may share physical machine, connection pathways and that is why the performance of each virtual machine can vary not only in time but may also depend on the "current" location (what physical machine it is currently running on) of virtual machine. Flexibility of public cloud is high and performance requirements can be changed very often (in days, hours, or even minutes).

\section{2) Service parameters}

Service parameters depend on the current hardware that the provider uses, and on virtualization parameters. When an application of one customer's virtual machine loads fully provided capacity of a virtual machine, there may be decreased capacities for virtual machines of other customers using the same physical machine. It is therefore important that the provider has set the virtualization conditions in a way that customers do "not interfere" each other. From the perspective of the provider it is of course advantageous to share a single physical machine by as many virtual machines as possible, but when the limits are set incorrectly, customers may experience performance drops of their individual virtual machines.

It is therefore appropriate to ask provider about performance guarantee parameters offered by virtual machines. These parameters can be expressed in different ways. Very precise measurable performance parameter for the virtual machine is the value of "CPU ready time" described in
[15]. This value indicates the response times of individual virtual machines. Nevertheless, the computing power is not the only indicator that should be monitored - for example, data storage and transmission networks are also important parameters to study before making final decision about a provider. "Disk Response Time" is for example a value, which indicates speed of storage system (it is affected by the length of storage request queue and disk array speed).

Sharing hardware resources may evoke in customers unpredictable performance of purchased virtual machines and it is therefore recommended to "test" the service before signing the contract. The test should take at least one week in full operation in order to determine whether the requirements for an information system with virtualized machines are met. When the provider is unwilling or unable to guarantee the performance parameters, it is advisable to ensure the "backdoor" in the form of rapid termination of the contract without additional charge. On the other hand, it is not particularly difficult to change the provider within IaaS (with respect to PaaS and SaaS services).

\section{3) Economic characteristics}

The price in case of public cloud is determined much more accurately and depends mostly on the number of machines purchased, the number of data transferred between could and the Internet and the size of the required disk space.

Global cloud providers like Amazon, Rackspace and Microsoft publish prices on their websites local and smaller providers especially in the Czech Republic do not publish prices because of their trade policies, but it can be assumed that prices will range between the prices of private cloud and those that are offered by large public cloud providers.

\section{PaaS}

\section{1) Service description}

The difficulty with choice of provider in this case lies in different databases that providers offer. They also vary in the available programming languages for writing applications and there are differences in provided API. For this reason, it is very difficult to move applications between different providers and there arise a dependency and selected provider. It is therefore important to select such a provider, who can be trusted in terms of stability, or the one who's API is compatible with any other alternative provider, so in case of problems it is possible to switch between providers(there exist kinds of API bridges for example "libcloud").

\section{2) Service parameters}

PaaS service parameters depend on the needs of the customer-developed applications. Performance in terms of processor and memory can be changed according to predefined virtual machines on offer. Provided is a virtual machine as well as in the case of IaaS but there is the difference in price offered for the machine, and there is preinstalled environment for running developed applications.

3) Economic characteristics

Payment model is similar to IaaS. Number of virtual 
machines used is charged as well as storage database (depending on usage - number of operations with database per mouth and amount of stored data) and network traffic (amount of data transferred between virtual machines and internet). Fees depend on the usage of PaaS services so the application design itself should take into account the very payment model. For example, database operations are charged separately. When designing a cloud application it is therefore necessary to focus on the most effective communication between application and database (to form query correctly and avoid unnecessary operations) so that the price per unit of time does not grow unnecessarily high because of poor program design. The situation is similar for transmission of data between cloud and the Internet.

E. SaaS

1) Service description

From the perspective of customer, the trust is the most important in the choice of the SaaS provider. The customer is going to be tightly bound to his provider, since provider manages customer's data. The problem is not only data security, but also in the ability to transfer data to an-other provider. Specific SaaS applications may store data to the database in unknown format, and it may be very difficult or even impossible to export data from database in certain portable format. This factor gives force to provider over the customer, thus it is important that the provider acts ethically and offers the service at adequate prices. The risk of provider bankruptcy or service termination must be considered, when selecting SaaS services as well.

\section{2) Service parameters}

Provider is responsible for service parameters and these are listed in the agreement between the provider and the customers - usually enclosed in the SLA. It is therefore quite important to monitor, whether the current wording of this agreement will ensure service that is available and fully functional.

\section{3) Economic characteristics}

Providers of SaaS very often chose "software leasing" as a payment model as mentioned in [16] because it is simple and easy to implement, transparent and predictable in terms of price for both provider and customer. The final price per month will vary depending on the functionality offered and mass recovery.

\section{DISCUSSION}

In the article were mentioned several topics around cloud type service selection and the most significant operational and economic risks connected to cloud services.

All facts stated in this article resulted from conversations between the author, providers of cloud services and customers of cloud services. The dialogue was led principally with business entities excluding government organizations. What concerns government and non-profit organizations the cloud computing is also quite appealing model of outsourcing and government can expect a similar ways of usage of publicly available cloud services.

All the participants were from the Czech Republic so the conclusion will be applicable for this region; however, there are some differences between countries and those may cause some issues with introduced technique in countries outside this region.

We cannot say with certainty that this article covered all significant risks of economical and operational nature, but the vast majority of most important questions that should be answered by a customer considering cloud services are mentioned in the article.

\section{Closure}

This article provided guidance for cloud service selection.

We presented the process and important standards related to decision making about cloud services taking into account specific needs of various customer groups. With help of above introduced process the management and ICT employees conducting a study of possible cloud usage may identify and focus on one specific class of cloud services and save time spent analyzing services that are unsuitable.

Furthermore, they need to make a market research to find a set of available cloud services.

Third step is to choose best fit from the set of cloud offers. To help with this step, the operational and economic risks were mentioned in the article.

\section{ACKNOWLEDGMENT}

The work on this article was supported by University of Economics in Prague internal grant agency (grant IG409061).

\section{REFERENCES}

[1] R. Buyya, C. S. Yeo, and S. Venugopal. Market-oriented cloud computing: Vision, hype, and reality for delivering it services as computing utilities. In Proc. The 10th IEEE International Conference on High Performance Computing and Communications (HPCC-08), Dalian, China, pp. 5-13, 2008.

[2] G. Briscoe and A. Marinos. Digital Ecosystems in the Clouds: Towards Community Cloud Computing In Proceedings of the 3rd IEEE International Conference on Digital Ecosystems and Technologies: (DEST 2009). pp. $103-108,2009$.

[3] A. Goldstein. Calculating the Cloud Determining the True Cost of Hosting Servers in the Cloud. NERCOMP 2010 Annual Conference. Educase, 2010.

[4] P. Doucek. Applied information management - Management reference model - security metrics. In Proc. 16th Conference on Interdisciplinary Information Management Talks (IDIMT 2008), Jindrichuv Hradec, , pp. 81-106, 2008.

[5] E.D. Hoadley, A. Laughton and J. Purcell. Resource Management: Guidelines For Managing In A Crisis. Journal of Business \& Economics Research. 7th ed., vol. 8.pp. 79-84, 2009.

[6] D. Linthicum. Selecting the right Cloud. InfoWorld Cloud Computing Deep Dive, pp. 2-9, 2009.

[7] P. Mell, and T. Grance. The NIST Definition of Cloud Computing Recommendations of the National Institute of Standards and Technology. The National Institute of Standards and Technology. 2011.

[8] J. Peng, X. Zhang, Z. Lei, B. Zhang, W. Zhang and Q. Li. Comparison of Several Cloud Computing Platforms. In Proceedings of the 2009 Second International Symposium on Information Science and 
Engineering (ISISE '09). IEEE Computer Society, Washington, DC, USA, pp. 23-27, 2009

[9] M. Skilton, P. Gordon. Cloud Buyers' Decision Tree. 2010. [Online] <http://www.opengroup.org/cloud/whitepapers/wp_cloud_dt/index.htm>

[10] W. Van Grembergen, Wim. Strategies for information technology governance. Hershey: Idea Group Pub., p. 390, 2003, ISBN 15-9140141-0.

[11] K. Dahbur, Mohammad, B., and Tarakji, A. B. A survey of risks, threats and vulnerabili-ties in cloud computing. In Proceedings of the 2011 International Conference on Intelligent Semantic Web-Services and Applications (New York, NY, USA, 2011), ISWSA '11, ACM, pp. 12:1-12:6, 2011

[12] T. Mather, S. Kumaraswamy, S. Latif. Cloud Security and Privacy: An Enterprise Perspective on Risks and Compliance. O'Reilly, Gravenstein, 2009.

[13] H. Wang, F. Liu, H. Liu. A Method of the Cloud Computing Security Management Risk Assessment. Advances in Intelligent and Soft Computing. (Springer Berlin / Heidelberg), pp 609-618. 2012.

[14] B. T. Ward, J. C. Sipior. The Internet jurisdiction risk of cloud computing. Information Systems Management, Vol. 27, Iss. 4.pp 334$339,2010$.

[15] T. Kellog. Esx guest capacity determination using guest ready-time metric as an indicator. In Int. CMG Conference (2008), Computer Measurement Group, pp. 465-474. 2008.

[16] OJALA, A. Comparison of different revenue models in saas. In Proceedings of 5th cloud computing and virtualization conference (2012), Global Science \& Technology Forum, pp. 120-123, 2012.

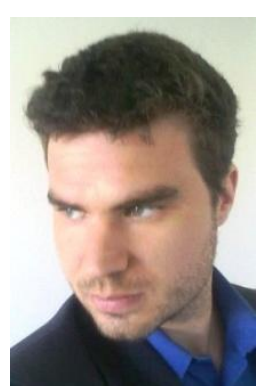

Jaromir Veber was born in Czech Republic in 1983. He accomplished master degree on Faculty of Electrical Engineering of Czech Technical University in Prague. He is currently studying doctoral study at Faculty of Informatics and Statistics of University of Economics in Prague with a focus on cloud computing, new technology and security. 